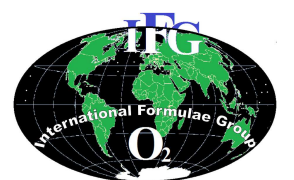

Available online at http://ajol.info/index.php/ijbcs

Int. J. Biol. Chem. Sci. 9(1): 35-47, February 2015

International Journal

of Biological and

Chemical Sciences

ISSN 1997-342X (Online), ISSN 1991-8631 (Print)

Original Paper

http://indexmedicus.afro.who.int

\title{
Botanical indices of ploidy levels in some African accessions of Oryza punctata Kotschy ex Steud
}

\author{
Chinyere Constance NWOKEOCHA
}

Obafemi Awolowo University, Institute of Natural History Museum, Ile-Ife, Nigeria. E-mail: chyabey27@yahoo.com; Tel: +234(0)8138338473

\begin{abstract}
Twenty-nine accessions of Oryza punctata Kotschy ex Steud, from local and other African habitats were studied to establish the attributes that can delineate the two ploidy levels based on agro-botanical, foliar epidermal and nodal anatomical characteristics. The diploid plants of $O$. punctata are early-maturing annuals with a small biomass, while the tetraploids are large, vigorous perennials. From the anatomical and morphological traits: the diploids have narrower leaves $(0.95-1.36 \mathrm{~cm}$ wide $)$ than the tetraploids $(1.9-2.3$ $\mathrm{cm})$; the culms of diploids are generally thin $(0.28-0.35 \mathrm{~cm}$ in diameter $)$ compared to the tetraploids $(0.37$ $0.57 \mathrm{~cm})$; the diploids have longer spikelets $(6.20-7.04 \mathrm{~mm})$ with length/breadth $>3$; longer awns $(52-86$ $\mathrm{mm}$ ) than the tetraploids $(20-31 \mathrm{~mm})$. The basal cells of microhairs in the diploids are about twice the length of apical cells, but only slightly longer than the apical cells in the tetraploids; prickle hairs are sparse in the intercostal zone of diploids, but abundant in tetraploids; short cells are paired and abundant in the intercostal zone of diploids, but sparse and solitary in the tetraploids. These indices delineated the ploidy levels and are reliable for use as identification aids.
\end{abstract}

(c) 2015 International Formulae Group. All rights reserved.

Keywords: Agro-botanical characteristics, diploid punctata, tetraploid punctata, delineation, identification aids.

\section{INTRODUCTION}

Sano (1980) compared the adaptive strategies in diploid and tetraploid forms of Oryza punctata Kotschy ex Steud. He reported that diploid strains had a high reproductive effort, well developed awns, low regeneration of excised stem segments, short anthers and a high degree of seed dormancy. The diploid strains were from open grasslands while the tetraploid strains were confined to the forest. Faluyi and Nwokeocha (1993) reported a widespread distribution of diploids and an overlap in the distribution of diploids and polyploids $(4 n=48)$ but with the polyploids spreading beyond the range of diploids. Watanabe et al. (1993) attributed the inability of diploid $O$. punctata to adapt to low light to the total amount of chlorohyll produced or the partitioning of the thylakoid protein into the core complex of photosystems II.

The taxonomic importance of cuticular features of plant surfaces had been revealed as early as 1965 by Stace. Srivastava (1978) described the leaf epidermis as the second most important character after cytology for 
solving taxonomic problems. For decades, anatomical and foliar epidermal features have been shown to possess great significance in the taxonomy of many taxa. Many of these attributes have been found to be diagnostic at generic or specific levels while others are important only in combination of other characters. This fact is illustrated in the works of Stace (1984), Ogundipe and Olatunji (1991), Nwokeocha (1996), Jayeola et al. (2001), Adedeji and Faluyi (2001), Adedeji and Illoh (2004), Kadiri et al. (2005), Ayodele and Olowokudejo (2006), Adedeji and Jewoola (2008), Ogie-Oda et al. (2010), Adedeji (2011), Munir et al. (2011), Adeniji and Ariwaodo (2012), Thakur and Patil (2014). The outcome of these works is a better understanding of the taxonomy of the taxa involved. These successes have been attributed to the fact that the features involved are subject to limited environmental variation and therefore are reliable.

The taxonomy and identification of the species in the $O$. officinalis complex, sometimes also referred to as $O$. latifolia complex, are difficult due to similar morphology and overlapping distribution of some of the species. The difference in ploidy levels of some species has been reported to add to the complexity (Faluyi and Nwokeocha, 1993; Bao et al., 2005). Also, the efficient utilization of rice genetic resources and efficient management of the germplasm collections rely on the correct identification of the germplasm (Ge et al., 2001; Li et al., 2001). This paper, therefore, investigated the agro-botanical and morphological characteristics, in addition to some nodal and foliar epidermal features, of the two ploidy levels of $O$. punctata. This was with a view to distinguishing between diploid and tetraploid O. punctata, which belong in the latifolia complex, and providing identification aids.

\section{MATERIALS AND METHODS}

Plant source

A total of 29 accessions of Oryza punctata, collected from the Genetic
Resources Unit of International Institute for Tropical Agriculture (IITA) and from our locality, as seen in Table 1, were studied. The plant materials collected (seeds and rootstocks) were grown in both screen house and field and were maintained for several years. Measurements of quantitative and qualitative morphological characters from culm, leaf, ligule, panicle, spikelet, awn, anther, pollen grain, among others, were taken for all the accessions based on the IBPGRIRRI Rice Advisory Committee (1980) Descriptors for Rice, Oryza sativa Linn.

\section{Foliar epidermal studies}

Mid portions of the abaxial and adaxial surfaces of leaf blade were scraped with sharp razor blade to prepare the epidermides according to Cutler (1978). The peels were decolourized in $5 \%$ solution of sodium hypochlorite (domestic bleach) for 30-60 minutes after which they were preserved in $50 \%$ ethanol. The cleared epidermal peels were stained in Alcian blue and counterstained in Toluidene blue to enhance contrast. To enhance the identification of cork and silica cells, epidermal peels were stained in $1 \%$ Sudan IV. Peels were mounted on microscope slides in $25 \%$ glycerol. Illustrations of epidermal features were made with the aid of Camera Lucida outfit at X25 and X40 objective powers. Measurements of cells, microhairs and stomata were made in ocular units and converted to micrometers. Stomata frequency per $\mathrm{mm}^{2}$ was determined.

\section{Nodal anatomical studies}

Transverse sections (T.S.) of the first three nodes above the ground were made on the Reichert sliding microtome at $15 \mu \mathrm{m}$. The sections were preserved in FAA (FormalinAcetic-Alcohol), stained in $1 \%$ aqueous solution of Safranin 'O', counter-stained in Alcian blue for 3-5 minutes, differentiated and dehydrated in different grades of concentration of alcohol up to $95 \%$ and mounted in DPX. Photomicrographs of the 
T.S. of nodes were taken under $\mathrm{X} 4$ objective power.

\section{Statistical analysis}

Quantitative data were analyzed using ANOVA with a significant level of $\mathrm{p}=0.05$.

\section{RESULTS \\ Habitat/Habit}

The local accessions were found on marshy ground, river beds of stagnant water except where man has interfered with the natural ecosystem as shown in Table 1. Two types of habit were encountered: diploids $(2 \mathrm{n}=24)$ which are annuals and tetraploids $(2 \mathrm{n}=48)$ which are perennials. The diploids are earlier maturing (98 - 105 days) than the tetraploids (124-148 days).

\section{Plant type}

Three major plant types were observed: the open, the erect and the spreading to procumbent. The open plant type, with culms angle c. $50^{\circ}$ comprises TOP 5702, TOP 14097 and TOP 14460, all diploids, whose culms are thin, heavy tillering with leaves long and narrow; the accessions from CAR and TOP 13596 from Tanzania whose culms are tall and fat, heavy tillering with leaves long and broads. The erect plant type (with culms angle c. $30^{\circ}$ ) comprises TOP 6788 and TOP 8222 which are low tillering with leaves long, broad and densely pubescent. The diploid TOP 13546, representing the third plant type, is fairly heavy tillered with thin culms which are between procumbent and prostrate at late vegetative phase. The length/breadth ratio of leaves separated the diploids from the tetraploids without overlap as shown in Table 2.

\section{Reproductive biology}

The result is shown in Table 3.

\section{Panicle}

The panicle type encountered in this study is open with loose ascending or spreading to descending primary branches. The length is variable among accessions but generally shorter in the diploid forms. The panicle density is also variable.

\section{Spikelet}

Shattering habit is present in all the accessions. The pericarp colour is brown; the stigma is dark-purple. Awn is present in all the accessions. The diploid 0. punctata has very long and broad spikelets (1:b ratio > 3) with very long awns while the tetraploids have long and very broad spikelets $(1: b$ ratio < 3 ) with short awns.

\section{Anther}

The anther colour is dirty-brown except in TOP 6788 and TOP 8222 which have milkcoloured anthers. The anther sizes among the accessions vary but of medium size.

\section{Pollen grain}

The pollen grain types/forms in all the accessions are of a typical grass: monoporate, spherical with thick exine which is raised around a circular pore; surface psilate or reticulate; of medium size. The size and sculpturing pattern under light microscope did not distinguish between diploid and tetraploid O. punctata.

\section{Foliar epidermal studies}

The result is shown in Tables 4 and 5, and Figure 1.

\section{Costal zone}

Long cells rectangular, many times longer than broad, cell walls markedly sinuous; papillae numerous per cell, of uniform size, circular and dome-shaped; short cells abundant in continuous rows; silica bodies cross-shaped (Oryza-type; according to Metcalfe, 1960), in pairs. Prickle hairs frequent, also on the margin of the leaf blade. Macrohair sparse to frequent in all, except TOP 6788 and TOP 8222, long and slender with large, raised base and pointed apex. Microhair and Stomata not seen.

\section{Intercostal zone}

Long cells rectangular, longer than broad, generally longer on abaxial than on the adaxial epidermides; cell walls markedly sinuous; papillae numerous per cell, rounded, small-sized and uniform on adaxial, a 
combination of small and largely inflated ones on abaxial epidermides; short cells sparse usually solitary in tetraploids, numerous and usually paired in diploids; stomata abundant, paracytic, 2 bands per intercostal region, 1 - 2 rows per band; subsidiary cells predominantly triangular in shape. Prickle hairs abundant in tetraploids but sparse in diploids. No macrohair was seen. Microhair numerous, bicellular, basal cells slightly longer than apical cells in tetraploids but about twice the length of apical cells in diploids

\section{Nodal anatomy}

The transverse section through the node in all accessions studied revealed the following general features as presented in Figure 2.

The epidermis composed of 1-layered cell, generally isodiametric, occasionally substended by 1-2 layered sclerenchyma cells. Outer ground tissue composed of 2 - 5 layers of parenchyma cells, occasionally lignified and sometimes intercepted by 4 - 8 layers of sclerenchyma cells extending to epidermis and traverses the entire inner ground tissue; small vascular bundles are embedded within the sclerenchyma at intervals. Inner ground tissue composed of bundles of primary vascular bundles surrounded by extraxylary fibres, smaller vascular bundles in-between primary vascular bundles. Towards central ground tissue is 1 circle of large vascular bundles surrounded by extraxylary fibres and 1 - 2 layers of sclerenchyma cells. Sclereids occasionally seen. Central ground tissue composed of schizogenous cells. Pith at node is solid.
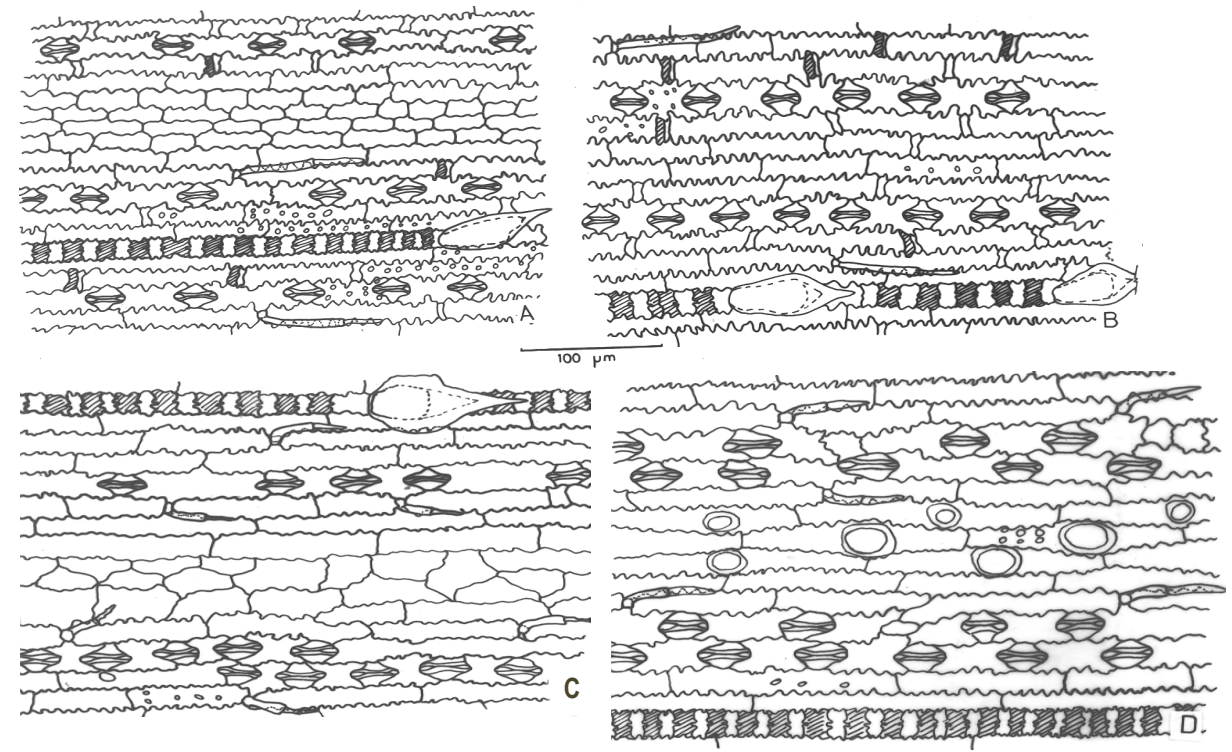

Figure 1: Representatives of Foliar Epidermal features of $O$. punctata studied. A: Adaxial of diploid; B: Abaxial of diploid; C: Adaxial of tetraploid, D: Abaxial of tetraploid. 

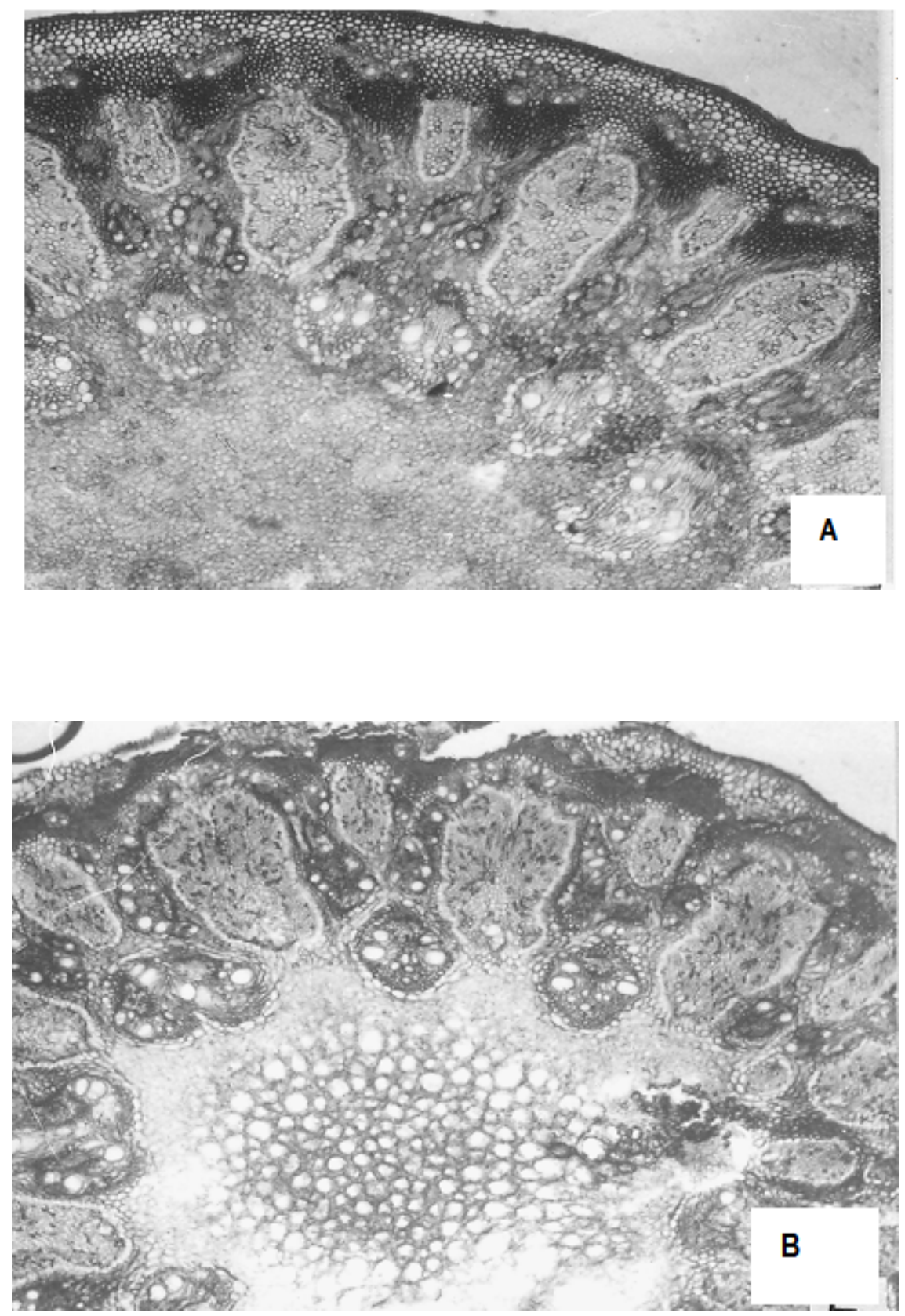

Figure 2: Representatives of Nodal Anatomical Features of O. punctata Species Studied. A. $2 \mathrm{n}=48$. B. $2 n=24$. 
Table 1: Accessions of Oryza punctata studied and their sources.

\begin{tabular}{|c|c|c|c|c|}
\hline $\begin{array}{l}\text { Accession } \\
\text { Number }\end{array}$ & Collector and Location & $\begin{array}{l}\text { Collection } \\
\text { Date }\end{array}$ & $\begin{array}{c}\text { Chromosome } \\
\text { Number }\end{array}$ & Description \\
\hline TOP $^{\mathrm{a}}$ IPETU & NWOKEOCHA: Ipetumodu & $28 / 9 / 90$ & 48 & \multirow{9}{*}{$\begin{array}{c}\text { Large Population on } \\
\text { marshy ground around poo } \\
\text { of water, close to human } \\
\text { habitation; open location. }\end{array}$} \\
\hline 01 & $7^{\circ} 30^{\prime \prime} \mathrm{N} 4^{\circ} 45^{\prime \prime E}$, Nigeria & $"$ & 48 & \\
\hline 02 & & " & 48 & \\
\hline 03 & & $"$ & 48 & \\
\hline 04 & & $"$ & 48 & \\
\hline 05 & & $"$ & 48 & \\
\hline 06 & & $"$ & 48 & \\
\hline 07 & & ” & 48 & \\
\hline 08 & & $"$ & 48 & \\
\hline TOP MORO & NWOKEOCHA: Moro & $28 / 9 / 90$ & 48 & \multirow{4}{*}{$\begin{array}{l}\text { Few stands on the bed of a } \\
\text { river, sandy soil, located } \\
\text { under shade of Alchornea } \\
\text { cordifolia }\end{array}$} \\
\hline 09 & 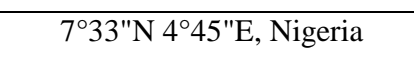 & $"$ & 48 & \\
\hline 10 & “ & $"$ & 48 & \\
\hline 11 & “ & $"$ & 48 & \\
\hline TOP RD7 ${ }^{\mathrm{F}}$ & FALUYI: RD $7^{\mathrm{F}}$ O.A.U. & $5 / 10 / 90$ & 48 & \multirow{4}{*}{$\begin{array}{l}\text { Along the valley of a river. } \\
\text { Location is swampy, open } \\
\text { in part, largely under } \\
\text { shade. }\end{array}$} \\
\hline 12 & Campus, Ile-Ife; & $"$ & 48 & \\
\hline 13 & $7^{\circ} 33^{\prime \prime} \mathrm{N} 4^{\circ} 31^{\prime \prime E}$, Nigeria & $"$ & & \\
\hline 14 & “ & $"$ & 48 & \\
\hline TOP SEKONA & NWOKEOCHA: Sekona & $12 / 10 / 90$ & 48 & \multirow{2}{*}{$\begin{array}{l}\text { Few stands on river bed, } \\
\text { road side. Location is open }\end{array}$} \\
\hline 15 & $7^{\circ} 33^{\prime \prime N} 4^{\circ} 38^{\prime \prime E}$, Nigeria & $"$ & & \\
\hline 16 & “ & & & \\
\hline TOP 8222 & 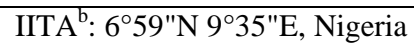 & $11 / 3 / 90$ & 48 & Wild on fallow land \\
\hline TOP 6788 & “ & & 48 & “ \\
\hline TOP 15114 & IITA: $4^{\circ} 22^{\prime \prime} \mathrm{N} 18^{\circ} 30^{\prime \prime} \mathrm{E}, \mathrm{CAR}^{\mathrm{C}}$ & $"$ & 48 & " \\
\hline TOP 15115 & IITA: $4^{\circ} 23^{\prime \prime N} 18^{\circ} 30^{\prime \prime E}$, CAR & $”$ & 48 & “ \\
\hline TOP 15116 & IITA: $6^{\circ} 53 " \mathrm{~N} 19^{\circ} 07^{\prime E}$, CAR & $"$ & 48 & $"$ \\
\hline TOP 15117 & IITA: $6^{\circ} 55^{\prime \prime N} 19^{\circ} 06^{\prime E}$, CAR & $"$ & 48 & $"$ \\
\hline TOP 15118 & IITA: $7^{\circ} 02^{\prime \prime N} 18^{\circ} 50 " \mathrm{E}, \mathrm{CAR}$ & $"$ & 48 & $"$ \\
\hline TOP 15119 & IITA: $4^{\circ} 20^{\prime \prime} \mathrm{N} 18^{\circ} 30^{\prime \prime E}$, CAR & $"$ & 48 & ” \\
\hline TOP 13596 & IITA:--------Tanzania & $"$ & 48 & -- \\
\hline TOP 13546 & IITA: ------- Tanzania & ” & 24 & -- \\
\hline TOP 5702 & IITA: $7^{\circ} 22 " \mathrm{~N} 7^{\circ} 50 " \mathrm{E}$, Nigeria & $"$ & 24 & Wild Vegetation \\
\hline TOP 14097 & IITA: ------- Tanzania & $"$ & 24 & -- \\
\hline TOP 14460 & IITA: ------- Nigeria & $"$ & 24 & Wild on fallow land \\
\hline
\end{tabular}


Table 2: Morphological features of the accessions of Oryza punctata studied.

\begin{tabular}{|c|c|c|c|c|c|c|c|c|c|}
\hline \multirow[t]{2}{*}{ Accession Number } & \multicolumn{2}{|c|}{$\begin{array}{c}\text { Flag leaf } \\
(\mathrm{cm})\end{array}$} & \multicolumn{2}{|c|}{$\begin{array}{l}\text { Leaf below } \\
(\mathrm{cm})\end{array}$} & \multirow{2}{*}{$\begin{array}{c}\begin{array}{c}\text { Culm Length } \\
(\mathrm{cm})\end{array} \\
\mathrm{l}\end{array}$} & \multirow{2}{*}{$\begin{array}{c}\begin{array}{c}\text { Culm Diameter } \\
(\mathbf{c m})\end{array} \\
\mathbf{b}\end{array}$} & \multirow[t]{2}{*}{ No. } & \multirow[t]{2}{*}{$\begin{array}{l}\text { Ligule length } \\
\text { (cm) }\end{array}$} & \multirow[t]{2}{*}{$\begin{array}{l}\text { Days to } \\
\text { heading* }\end{array}$} \\
\hline & l & $\mathbf{b}$ & l & b & & & & & \\
\hline Ipetu-Moro & $27.10 \pm 1.36$ & $1.90 \pm 0.05$ & $41.10 \pm 3.15$ & $1.96 \pm 0.09$ & $152.02 \pm 1.24$ & $0.42 \pm 0.02$ & 38 & $6.58 \pm 0.03$ & 145 \\
\hline $\operatorname{Rd} 7^{\mathrm{F}}$ & $25.80 \pm 2.26$ & $1.80 \pm 0.03$ & $43.80 \pm 3.04$ & $1.90 \pm 0.06$ & $1.56 .18 \pm 1.42$ & $0.45 \pm 0.03$ & 27 & $5.80 \pm 0.02$ & 145 \\
\hline Sekona & $23.70 \pm 2.22$ & $1.80 \pm 0.04$ & $36.22 \pm 1.57$ & 1.96 .004 & $151.90 \pm 1.50$ & $0.43 \pm 0.03$ & 34 & $6.62 \pm 0.04$ & 148 \\
\hline 8222 & $36.50 \pm 2.21$ & $2.60 \pm 0.02$ & $44.96 \pm 2.90$ & $1.96 \pm 0.09$ & $1.36 .96 \pm 0.65$ & $0.36 \pm 0.02$ & 17 & $2.10 \pm 0.02$ & 124 \\
\hline 6788 & $31.00 \pm 2.24$ & $2.50 \pm 0.03$ & $45.52 \pm 1.87$ & $1.94 \pm 0.05$ & $131.48 \pm 0.74$ & $0.35 \pm 0.02$ & 13 & $2.20 \pm 0.02$ & 126 \\
\hline 15114 & $17.80 \pm 1.93$ & $1.67 \pm 0.04$ & $35.84 \pm 2.67$ & $1.84 \pm 0.05$ & $72.50 \pm 1.48$ & $0.35 \pm 0.08$ & 7 & $5.40 \pm 0.04$ & 141 \\
\hline 15115 & $25.70 \pm 2.08$ & $2.50 \pm 0.10$ & $51.22 \pm 3.50$ & $1.94 \pm 0.08$ & $66.87 \pm 1.60$ & $0.43 \pm 0.03$ & 19 & $3.25 \pm 0.04$ & 132 \\
\hline 15116 & $17.30 \pm 1.97$ & $2.00 \pm 0.09$ & $37.40 \pm 2.76$ & $2.00 \pm 0.05$ & $101.77 \pm 1.48$ & $0.43 \pm 0.03$ & 12 & $4.51 \pm 0.03$ & 145 \\
\hline 15117 & $20.70 \pm 1.95$ & $2.20 \pm 0.06$ & $39.32 \pm 2.93$ & $2.12 \pm 0.13$ & $129.80 \pm 1.02$ & $0.43 \pm 0.03$ & 14 & $5.22 \pm 0.03$ & 137 \\
\hline 15118 & $26.60 \pm 2.20$ & $2.00 \pm 0.04$ & $41.36 \pm 2.53$ & $2.06 \pm 0.06$ & $114.00 \pm 1.06$ & $0.42 \pm 0.03$ & 19 & $5.51 \pm 0.02$ & 132 \\
\hline 15119 & $24.70 \pm 1.86$ & $2.20 \pm 0.11$ & $44.22 \pm 2.60$ & $1.92 \pm 0.07$ & $134.70 \pm 1.31$ & $0.50 \pm 0.02$ & 21 & $5.10 \pm 0.03$ & 145 \\
\hline 13596 & $23.70 \pm 2.05$ & $1.80 \pm 0.08$ & $38.12 \pm 1.94$ & $1.96 \pm 0.08$ & $106.50 \pm 2.21$ & $0.47 \pm 0.03$ & 11 & $6.15 \pm 0.05$ & 136 \\
\hline 13546 & $32.20 \pm 2.15$ & $1.50 \pm 0.03$ & $44.56 \pm 2.16$ & $1.32 \pm 0.04$ & $104.60 \pm 1.35$ & $0.32 \pm 0.03$ & 15 & $6.20 \pm 0.04$ & 139 \\
\hline 5702 & $35.10 \pm 2.08$ & $1.30 \pm 0.02$ & $52.34 \pm 2.09$ & $1.02 \pm 0.04$ & $86.35 \pm 1.09$ & $0.32 \pm 0.02$ & 25 & $3.53 \pm 0.03$ & 105 \\
\hline 14097 & $36.50 \pm 2.17$ & $1.30 \pm 0.02$ & $48.22 \pm 0.90$ & $0.99 \pm 0.03$ & $87.10 \pm 1.12$ & $0.30 \pm 0.02$ & 25 & $4.10 \pm 0.04$ & 98 \\
\hline 14460 & $37.40 \pm 2.19$ & $1.30 \pm 0.03$ & $43.72 \pm 1.03$ & $0.97 \pm 0.02$ & $72.10 \pm 0.93$ & $0.30 \pm 0.02$ & 16 & $3.70 \pm 0.03$ & 100 \\
\hline
\end{tabular}


Table 3: Floral attributes of the accessions of Oryza punctata studied.

\begin{tabular}{|c|c|c|c|c|c|c|c|c|c|c|c|}
\hline $\begin{array}{l}\text { Accession } \\
\text { Number }\end{array}$ & $\begin{array}{c}\text { Panicle } \\
\text { Length* } \\
\text { (cm) }\end{array}$ & $\begin{array}{l}\text { Panicle } \\
\text { Density }^{\text {a }}\end{array}$ & $\begin{array}{l}\text { Number of } \\
\text { Spikelets }\end{array}$ & $\begin{array}{c}\text { Awn } \\
\text { length* } \\
(\mathbf{m m})\end{array}$ & $\begin{array}{c}\text { Grain Size } \\
\text { length * } \\
\text { (cm) }\end{array}$ & $\begin{array}{c}\text { Grain Size } \\
\text { breadth * } \\
(\mathrm{cm})\end{array}$ & $\begin{array}{c}\text { Grain } \\
\text { Mean } \\
\text { l:b ratio }\end{array}$ & $\begin{array}{c}\text { Seed Set } \\
(\%)\end{array}$ & $\begin{array}{c}\text { Pollen } \\
\text { Fertility } \\
(\%)\end{array}$ & $\begin{array}{c}\text { Anther } \\
\text { length* } \\
(\mathrm{mm})\end{array}$ & $\begin{array}{c}\text { Pollen } \\
\text { Grain } \\
\text { size }^{* *}(\mu \mathrm{m})\end{array}$ \\
\hline Moro & $27.9 \pm 1.01$ & 1.53 & 240 & $21.50 \pm 0.50$ & $6.20 \pm 0.11$ & $2.25 \pm 0.04$ & 2.71 & 80.0 & 75.3 & $2.44 \pm 0.03$ & $43.81 \pm 0.35$ \\
\hline Ipetu & $28.0 \pm 1.04$ & 1.52 & 193 & $21.70 \pm 0.65$ & $6.20 \pm 0.11$ & $2.25 \pm 0.03$ & 2.76 & 79.8 & 73.4 & $2.42 \pm 0.02$ & $43.73 \pm 0.41$ \\
\hline $\operatorname{Rd} 7^{\mathrm{F}}$ & $27.8 \pm 0.66$ & 1.66 & 260 & $21.80 \pm 0.77$ & $5.75 \pm 0.11$ & $1.98 \pm 0.04$ & 2.90 & 80.8 & 96.1 & $1.84 \pm 0.03$ & $38.54 \pm 0.25$ \\
\hline Sekona & $21.5 \pm 0.50$ & 1.55 & 117 & $20.20 \pm 0.70$ & $5.78 \pm 0.11$ & $2.05 \pm 0.03$ & 2.82 & 76.1 & 79.4 & $1.75 \pm 0.03$ & $39.15 \pm 0.22$ \\
\hline 8222 & $26.1 \pm 0.59$ & 1.40 & 167 & $25.90 \pm 0.75$ & $6.49 \pm 0.09$ & $2.31 \pm 0.04$ & 2.81 & 85.6 & 92.1 & $1.24 \pm 0.02$ & $36.85 \pm 0.54$ \\
\hline 6788 & $20.1 \pm 0.54$ & 1.21 & 93 & $26.60 \pm 1.04$ & $6.11 \pm 0.07$ & $2.32 \pm 0.04$ & 2.63 & 84.9 & 96.5 & $1.74 \pm 0.03$ & $35.54 \pm 0.25$ \\
\hline 15114 & $20.5 \pm 0.60$ & 1.05 & 82 & $21.70 \pm 0.67$ & $5.72 \pm 0.07$ & $1.92 \pm 0.03$ & 2.98 & 87.8 & 95.9 & $2.30 \pm 0.02$ & $48.20 \pm 0.54$ \\
\hline 15115 & $21.6 \pm 0.49$ & 1.06 & 95 & $28.10 \pm 0.88$ & $5.38 \pm 0.10$ & $1.91 \pm 0.07$ & 2.82 & 92.2 & 97.3 & $2.07 \pm 0.02$ & $37.40 \pm 0.30$ \\
\hline 15116 & $22.8 \pm 0.51$ & 1.27 & 78 & $22.90 \pm 0.72$ & $5.78 \pm 0.09$ & $1.98 \pm 0.04$ & 2.92 & 65.4 & 93.1 & $2.23 \pm 0.02$ & $37.40 \pm 0.40$ \\
\hline 15117 & $27.7 \pm 0.54$ & 1.24 & 156 & $29.10 \pm 0.95$ & $5.62 \pm 0.05$ & $2.00 \pm 0.05$ & 2.81 & 91.0 & 92.1 & $2.19 \pm 0.02$ & $47.33 \pm 0.46$ \\
\hline 15118 & $23.9 \pm 0.49$ & 1.13 & 123 & $26.10 \pm 0.93$ & $5.91 \pm 0.06$ & $2.00 \pm 0.03$ & 2.96 & 87.2 & 91.2 & $2.14 \pm 0.02$ & $35.21 \pm 0.30$ \\
\hline 15119 & $22.3 \pm 0.52$ & 0.74 & 47 & $25.70 \pm 0.83$ & $5.68 \pm 0.06$ & $1.92 \pm 0.04$ & 2.96 & 78.7 & 94.9 & $2.35 \pm 0.03$ & $39.35 \pm 0.19$ \\
\hline 13596 & $25.4 \pm 0.51$ & 1.27 & 144 & $29.80 \pm 0.93$ & $5.62 \pm 0.05$ & $2.00 \pm 0.02$ & 2.81 & 81.9 & 95.3 & $1.95 \pm 0.02$ & $41.03 \pm 0.31$ \\
\hline 13546 & $21.4 \pm 0.54$ & 1.28 & 107 & $54.60 \pm 2.79$ & $6.27 \pm 0.09$ & $2.08 \pm 0.03$ & 3.01 & 87.9 & 92.2 & $2.00 \pm 0.03$ & $36.43 \pm 0.21$ \\
\hline 5702 & $20.5 \pm 0.52$ & 0.93 & 56 & $78.70 \pm 2.28$ & $6.98 \pm 0.06$ & $2.09 \pm 0.02$ & 3.34 & 85.7 & 97.6 & $1.60 \pm 0.02$ & $32.58 \pm 0.13$ \\
\hline 14097 & $16.7 \pm 0.50$ & 1.13 & 54 & $83.50 \pm 2.74$ & $6.87 \pm 0.05$ & $2.13 \pm 0.03$ & 3.22 & 85.0 & 93.3 & $1.74 \pm 0.03$ & $36.48 \pm 0.13$ \\
\hline 14460 & $15.2 \pm 0.55$ & 1.12 & 59 & $63.40 \pm 1.85$ & $6.79 \pm 0.06$ & $2.16 \pm 0.03$ & 3.14 & 83.1 & 92.5 & $1.84 \pm 0.03$ & $35.42 \pm 0.12$ \\
\hline
\end{tabular}

a - computed as a ratio of total spikelets to total length of radius and primary branches (Futsuhara et al., 1979).

- length, b- breadth

* - Tabulated values are Mean \pm Standard Error of 20 measurements; Significant level of $\mathrm{p}=0.05$.

** - Tabulated values are Mean \pm Standard Error of 100 measurements 
Table 4: Foliar epidermal features of the accessions of Oryza punctata studied.

\begin{tabular}{|c|c|c|c|c|c|c|c|c|c|c|}
\hline \multirow[t]{3}{*}{ Accession Number } & \multicolumn{2}{|c|}{ Stomata Frequency $\left(\mathrm{mm}^{2}\right)^{* *}$} & \multicolumn{4}{|c|}{ Stomata Size $(\mu \mathrm{m})^{*}$} & \multicolumn{4}{|c|}{ Long Cell Size $(\mu \mathrm{m})^{*}$} \\
\hline & \multirow[b]{2}{*}{ Adaxial } & \multirow[b]{2}{*}{ Abaxial } & \multicolumn{2}{|c|}{ Adaxial } & \multicolumn{2}{|c|}{ Abaxial } & \multicolumn{2}{|c|}{ Adaxial } & \multicolumn{2}{|c|}{ Abaxial } \\
\hline & & & 1 & $\mathbf{b}$ & l & $\mathbf{b}$ & $\mathbf{I}$ & $\mathbf{b}$ & 1 & $\mathbf{b}$ \\
\hline Ipetu-Moro & 145.18 & 209.63 & $22.25 \pm 0.59$ & $3.75 \pm 0.32$ & $28.00 \pm 0.38$ & $3.50 \pm 0.36$ & $82.50 \pm 3.94$ & $18.00 \pm 1.28$ & $87.50 \pm 3.97$ & $15.25 \pm 0.58$ \\
\hline $\operatorname{Rd} 7^{\mathrm{F}}$ & 147.59 & 173.49 & $24.00 \pm 0.67$ & $3.75 \pm 0.37$ & $28.50 \pm 0.40$ & $4.00 \pm 0.40$ & $91.00 \pm 4.04$ & $17.75 \pm 0.87$ & $97.78 \pm 3.76$ & $16.40 \pm 0.57$ \\
\hline Sekona & 148.79 & 216.26 & $21.00 \pm 0.55$ & $3.63 \pm 0.34$ & $23.50 \pm 0.36$ & $3.50 \pm 0.40$ & $80.00 \pm 4.12$ & $17.50 \pm 0.00$ & $122.50 \pm 6.40$ & $14.50 \pm 0.62$ \\
\hline 8222 & 118.07 & 163.85 & $23.25 \pm 0.28$ & $2.88 \pm 0.19$ & $25.88 \pm 0.45$ & $4.75 \pm 0.25$ & $74.00 \pm 5.06$ & $22.00 \pm 0.83$ & $77.50 \pm 3.94$ & $18.25 \pm 0.38$ \\
\hline 6788 & 118.67 & 189.76 & $24.00 \pm 0.64$ & $3.50 \pm 0.31$ & $25.25 \pm 0.55$ & $3.88 \pm 0.29$ & $61.50 \pm 2.14$ & $21.25 \pm 0.67$ & $78.50 \pm 2.91$ & $20.00 \pm 0.64$ \\
\hline 15114 & 152.83 & 185.54 & $22.13 \pm 0.38$ & $3.38 \pm 0.32$ & $25.63 \pm 0.33$ & $4.00 \pm 0.40$ & $91.95 \pm 2.52$ & $18.00 \pm 0.06$ & $90.25 \pm 3.62$ & $16.50 \pm 0.76$ \\
\hline 15115 & 156.62 & 297.83 & $22.50 \pm 0.56$ & $3.38 \pm 0.32$ & $26.00 \pm 0.41$ & $4.38 \pm 0.33$ & $101.50 \pm 2.74$ & $21.50 \pm 0.92$ & $112.00 \pm 4.11$ & $18.00 \pm 0.62$ \\
\hline 15116 & 125.30 & 217.88 & $21.75 \pm 0.50$ & $3.38 \pm 0.37$ & $23.63 \pm 0.39$ & $4.00 \pm 0.45$ & $85.50 \pm 4.58$ & $19.50 \pm 1.04$ & $86.00 \pm 4.46$ & $22.00 \pm 0.72$ \\
\hline 15117 & 163.25 & 172.29 & $22.13 \pm 0.48$ & $4.50 \pm 0.27$ & $25.50 \pm 0.53$ & $3.25 \pm 0.33$ & $110.50 \pm 4.54$ & $17.00 \pm 0.06$ & $103.50 \pm 5.86$ & $19.00 \pm 0.76$ \\
\hline 15118 & 153.61 & 181.92 & $23.75 \pm 0.32$ & $2.88 \pm 0.26$ & $26.25 \pm 0.37$ & $4.38 \pm 0.20$ & $95.50 \pm 3.97$ & $23.50 \pm 0.40$ & $85.75 \pm 5.45$ & $17.75 \pm 0.44$ \\
\hline 15119 & 170.48 & 180.12 & $22.13 \pm 0.37$ & $2.88 \pm 0.26$ & $26.88 \pm 0.68$ & $2.75 \pm 0.16$ & $89.00 \pm 5.27$ & $19.00 \pm 0.55$ & $92.50 \pm 4.12$ & $20.75 \pm 0.38$ \\
\hline 13596 & 136.56 & 221.08 & $24.63 \pm 0.53$ & $4.50 \pm 0.28$ & $26.00 \pm 0.40$ & $3.38 \pm 0.37$ & $70.00 \pm 1.97$ & $17.75 \pm 0.88$ & $84.73 \pm 4.60$ & $16.25 \pm 0.42$ \\
\hline 13546 & 181.30 & 228.91 & $23.87 \pm 0.47$ & $4.00 \pm 0.36$ & $25.75 \pm 0.33$ & $3.75 \pm 0.35$ & $90.75 \pm 4.86$ & $18.25 \pm 0.75$ & $102.50 \pm 4.36$ & $20.25 \pm 0.58$ \\
\hline 5702 & 178.91 & 182.53 & $24.50 \pm 0.48$ & $4.75 \pm 0.31$ & $27.50 \pm 0.26$ & $4.38 \pm 0.28$ & $77.25 \pm 5.18$ & $18.00 \pm 0.50$ & $84.25 \pm 3.42$ & $21.25 \pm 0.67$ \\
\hline 14097 & 187.95 & 252.41 & $24.00 \pm 0.76$ & $4.00 \pm 0.25$ & $23.25 \pm 0.38$ & $3.13 \pm 0.20$ & $82.50 \pm 5.28$ & $20.50 \pm 0.62$ & $87.50 \pm 2.86$ & $16.75 \pm 0.65$ \\
\hline 14460 & 184.93 & 239.15 & $23.13 \pm 0.69$ & $4.25 \pm 0.33$ & $20.88 \pm 0.75$ & $3.88 \pm 0.34$ & $103.50 \pm 4.41$ & $16.25 \pm 0.41$ & $113.50 \pm 6.20$ & $11.75 \pm 0.53$ \\
\hline
\end{tabular}

1 - length, b- breadth

* - Tabulated values are Mean \pm Standard Error of 100 measurements

** - Tabulated values are Mean \pm Standard Error of 100 measurements; Significant level of $\mathrm{p}=0.05$. 
C. C. NWOKEOCHA / Int. J. Biol. Chem. Sci. 9(1): 35-47, 2015

Table 5: Length and breadth $(\mu \mathrm{m})$ of the microhairs in the accessions of Oryza punctata studied.

\begin{tabular}{|c|c|c|c|c|c|c|c|c|c|c|}
\hline \multirow[t]{2}{*}{ Accession } & \multicolumn{3}{|c|}{ Adaxial } & \multicolumn{3}{|c|}{ Abaxial } & \multicolumn{2}{|c|}{ Total Length } & \multicolumn{2}{|c|}{ Basal (l):Apical (l) ratio } \\
\hline & $\begin{array}{c}\text { Basal Cell } \\
\text { (l) }\end{array}$ & $\begin{array}{c}\text { Apical Cell } \\
\text { (l) }\end{array}$ & $\begin{array}{c}\text { Max. } \\
\text { breadth }\end{array}$ & $\begin{array}{c}\text { Basal Cell } \\
\text { (l) }\end{array}$ & $\begin{array}{c}\text { Apical Cell } \\
\text { (l) }\end{array}$ & $\begin{array}{c}\text { Max. } \\
\text { breadth }\end{array}$ & Adaxial & Abaxial & Adaxial & Abaxial \\
\hline Ipetu-Moro & $33.10 \pm 1.16$ & $25.50 \pm 0.72$ & $5.13 \pm 0.12$ & $30.00 \pm 0.52$ & $26.60 \pm 0.64$ & $5.13 \pm 0.12$ & $58.60 \pm 1.88$ & $56.60 \pm 1.16$ & 1.298 & 1.128 \\
\hline $\operatorname{Rd} 7^{\mathrm{F}}$ & $30.95 \pm 0.91$ & $25.50 \pm 0.38$ & $5.00 \pm 0.00$ & $31.50 \pm 0.76$ & $26.75 \pm 0.38$ & $5.98 \pm 0.24$ & $56.25 \pm 1.25$ & $58.25 \pm 1.14$ & 1.206 & 1.178 \\
\hline Sekona & $28.00 \pm 0.62$ & $25.75 \pm 0.65$ & $5.00 \pm 0.00$ & $37.00 \pm 0.89$ & $32.25 \pm 0.87$ & $5.20 \pm 0.27$ & $53.75 \pm 1.27$ & $69.25 \pm 1.76$ & 1.087 & 1.147 \\
\hline 8222 & $32.00 \pm 0.72$ & $24.25 \pm 0.65$ & $5.06 \pm 0.07$ & $33.00 \pm 0.89$ & $25.75 \pm 0.83$ & $5.50 \pm 0.23$ & $56.25 \pm 1.37$ & $58.75 \pm 1.73$ & 1.320 & 1.282 \\
\hline 6788 & $32.50 \pm 0.83$ & $21.83 \pm 0.87$ & $5.18 \pm 0.09$ & $33.50 \pm 0.76$ & $23.50 \pm 0.55$ & $5.45 \pm 0.16$ & $54.33 \pm 1.70$ & $57.00 \pm 1.31$ & 1.489 & 1.426 \\
\hline 15114 & $38.00 \pm 1.22$ & $29.75 \pm 0.69$ & $5.00 \pm 0.00$ & $31.00 \pm 0.55$ & $25.50 \pm 0.62$ & $5.00 \pm 0.00$ & $67.75 \pm 1.92$ & $56.50 \pm 1.17$ & 1.277 & 1.216 \\
\hline 15115 & $32.00 \pm 0.82$ & $26.25 \pm 0.85$ & $5.00 \pm 0.20$ & $33.50 \pm 0.76$ & $31.00 \pm 1.00$ & $4.88 \pm 0.08$ & $58.25 \pm 1.67$ & $64.50 \pm 1.76$ & 1.219 & 1.081 \\
\hline 15116 & $24.00 \pm 0.36$ & $21.63 \pm 0.75$ & $5.00 \pm 0.00$ & $25.50 \pm 0.97$ & $24.45 \pm 0.66$ & $5.00 \pm 0.00$ & $45.63 \pm 1.11$ & $49.95 \pm 1.63$ & 1.110 & 1.043 \\
\hline 15117 & $30.75 \pm 0.75$ & $25.00 \pm 0.98$ & $4.95 \pm 0.07$ & $32.00 \pm 0.89$ & $28.00 \pm 0.62$ & $5.25 \pm 0.16$ & $55.75 \pm 1.73$ & $60.00 \pm 1.52$ & 1.230 & 1.143 \\
\hline 15118 & $33.50 \pm 1.06$ & $25.68 \pm 1 / 28$ & $5.00 \pm 0.00$ & $32.50 \pm 0.64$ & $25.50 \pm 0.50$ & $5.00 \pm 0.00$ & $59.18 \pm 2.34$ & $58.00 \pm 1.14$ & 1.305 & 1.275 \\
\hline 15119 & $29.50 \pm 0.81$ & $30.25 \pm 1.14$ & $5.00 \pm 0.00$ & $30.50 \pm 0.72$ & $31.25 \pm 1.13$ & $5.63 \pm 0.19$ & $59.75 \pm 1.96$ & $61.75 \pm 1.86$ & 0.975 & 0.976 \\
\hline 13596 & $38.00 \pm 0.81$ & $24.50 \pm 1.43$ & $5.13 \pm 0.29$ & $32.50 \pm 0.83$ & $33.00 \pm 0.72$ & $5.00 \pm 0.18$ & $62.50 \pm 2.25$ & $65.50 \pm 1.56$ & 1.551 & 0.985 \\
\hline 13546 & $50.45 \pm 1.31$ & $25.50 \pm 110$ & $5.00 \pm 0.00$ & $52.25 \pm 1.08$ & $26.75 \pm 0.79$ & $5.13 \pm 0.12$ & $75.95 \pm 2.42$ & $79.00 \pm 1.88$ & 1.978 & 1.953 \\
\hline 5702 & $43.10 \pm 1.22$ & $24.50 \pm 0.81$ & $4.95 \pm 0.05$ & $52.25 \pm 0.75$ & $31.25 \pm 1.67$ & $5.00 \pm 0.00$ & $67.50 \pm 2.04$ & $83.50 \pm 2.42$ & 1.755 & 1.736 \\
\hline 14097 & $40.00 \pm 1.78$ & $22.75 \pm 1.08$ & $4.95 \pm 0.00$ & $42.00 \pm 1.28$ & $23.75 \pm 1.35$ & $5.00 \pm 0.00$ & $62.75 \pm 2.87$ & $65.75 \pm 2.63$ & 1.758 & 1.768 \\
\hline 14460 & $40.00 \pm 1.05$ & $23.50 \pm 0.85$ & $4.88 \pm 0.12$ & $39.00 \pm 0.66$ & $25.00 \pm 0.61$ & $5.00 \pm 0.00$ & $63.50 \pm 1.90$ & $64.00 \pm 1.28$ & 1.702 & 1.560 \\
\hline
\end{tabular}

$1=$ length
Tabulated values are Mean \pm Standard Error of 20 measurements. 


\section{DISCUSSION}

The features of both diploid and tetraploid $O$. punctata revealed by this investigation agree with the report of Sano (1980) except for the anther length which was not significantly different between the two ploidy levels and the significantly taller polyploids found in this work. It was found that anther sizes are reliable only when they are measured at a uniform growth stage and that dehisced anthers are generally shorter than undehisced ones. The anthers measured in this study were those subtending primary branches whose spikelets had just bloomed but undehisced.

The diploids have longer spikelets (with length/breadth > 3), longer awns and narrower leaves than the tetraploids. Also the culms of diploids are generally thin as compared to those of tetraploids which are thicker. These morphological attributes form the field first aid to the rice collector before any laboratory examination.

The leaf epidermis in the rice under study, as in some other grasses, is demarcated into costal and intercostal zones within which the epidermal features are uniquely arranged and distributed so that certain features could be referred to as 'grass-type' or 'oryza-type'. This is supported by Metcalfe (1960) classification and agrees with the findings of Ogie-Oda et al. (2010). Of all the epidermal characters studied, the length of microhair, abundance of short cells and prickle hairs on intercostal zone showed significant differences between the diploid and tetraploid $O$. punctata. The basal cells of the microhair are about twice the length of apical cells in diploids contrary to the situation in tetraploid forms. Added to these are the abundance of short cells mostly paired in the intercostal zone of all the diploid forms but sparse and mostly solitary in tetraploids; and the prickle hairs which were abundant in the intercostal zone of the tetrapolids but very sparse or none seen in diploids. The above characters have delineated the diploid from the tetraploid $O$. punctata studied. Adeniji and Ariwaodo
(2012) elucidated the taxonomic importance of foliar epidermal features of the genus Pericopsis (Papilionaceae) in Nigeria and presented a complimentary data which would aid identification of the species even when only leaf fragments are available. By comparism, the diagonistic features of the leaf epidermis in the present study revealed that even when only leaf fragments of $O$. punctata are available, the ploidy level could still be determined. These identification aids or indices are easily accessible, visible, constant and consistent. They are taxonomically important, as they would facilitate and fasttrack the efficient utilization and management of rice genetic resources as suggested by Ge et al. (2001) and also enhance the understanding of the taxonomy of the species complex $(O$. latifolia complex).

Other characters, like long cell length and breadth; stomata type, length and breadth, shapes of the subsidiary cells; macrohairs and shape of the silica bodies, anther length, etc, showed slight variation among the accessions studied but could not reliably distinguish diploids from tetraploidy $O$. punctata. The features of the transverse section of the node also showed no significant variations among the accessions and between the two ploidy levels. Also the nodal anatomical features, especially the supporting tissues (collenchyma, sclerenchyma) did not correlate with the ploidy levels and did not support the plant types observed.

\section{Conclusion}

Several indices involving the habit, leaf breadth, culm diameter, awn length, microhair, short cells and prickle hair abundance, and spikelet length/breath ratio, have been established. These indices are reliable because they are subject to very limited environmental variation and they provide instant identification aids for the rice collector. They also serve as a base for germplasm cataloguing for future research and utilization. 


\section{REFERENCES}

Adedeji O, Faluyi JO. 2001. Foliar epidermal studies of thirty-five accessions of Panicum maximum Jacq in Nigeria. New Botanist., XXVIII: 145-167.

Adedeji O, Illoh HC. 2004. Comparative foliar anatomy of ten species in the Genus Hibiscus 6Linn. in Nigeria. New Botanist., XXXI: $147-180$.

Adedeji O, Jewoola OA. 2008. Importance of leaf epidermal characters in the Asteraceae family. Not. Bot. Hort. Agrobot., 36(2): 7- 16.

Adedeji O. 2011. Systematic significance of trichomes and foliar epidermal morphology in the species of Stachytarpheta Vahl.(Verbenaceae) from Nigeria. Thaiszia J. Bot., 22: 1-31.

Adeniji KA, Ariwaodo JO. 2012. Comparative foliar epidermal studies of genus Pericopsis (Papilionaceae) in Nigeria. Phytologia Balcanica, 18(1): 37-41.

Ayodele AE, Olowokudejo JD. 2006. The family Polygonaceae in West Africa: Taxonomic importance of leaf epidermal characters. South African Journal of Botany, 72: 442- 459.

Bao Y, Lu BR, Ge S. 2005. Identification of genomic constitutions of Oryza species with the $\mathrm{B}$ and $\mathrm{C}$ genomes by the PCRRFLP method. Genetic Resources and Crop Evolution., 52: 69-76.

Cutler DF. 1978. Applied Plant Anatomy (1 ${ }^{\text {st }}$ edn). Longman Inc.: New York; 5-6.

Faluyi JO, Nwokeocha CC. 1993. Occurrence and distribution of ploidy levels of Oryza punctata Kotschy ex Steud. in Africa. Feddes Repertorium, 104(3-4): 215-226.

Futsuhara Kondo S, Kitano H, Mii M. 1979. Genetical studies on dense and lax panicles in rice. I. character expression and mode or Lax panicle Rice. Japan J. Bread., 29(2): 151-155.

Ge S, Sang T, Lu BR, Hong DY. 2001. Rapid and reliable identification of rice gonomes by RFLP analysis of PCR- amplified Adh genes. Genome, 44: 1136-1142.

IBPGR-IRRI Rice Advisory Committee. 1980. Descriptors for Rice (Oryza sativa Linn.). IBPGR-IRRI Rice Advisory Committee.

Jayeola AA, Thorpe IR, Adenegan TA. 2001. Macromorphological and micromorphological studies of the West African Rhizophora L. Feddes Repertorium, 112(5-6): 349-356.

Kadiri AB, Olookudejo D, Ogundipe OT. 2005. Some aspects of foliar epidermal morphology of Cylicodicus gabunnesis (Taub) Harms (Mimosaceae). Journal of Science Research, 10: 33-38.

Li CB, Zang DM, Ge S, Lu BR, Hong DY. 2001. Identification of genome constitution of Oryza malampuzhaensis, O. minuta and $O$. punctata by multicolor genomic in situ hybridization. Theoretical and Applied Genetics, 103: 197-203.

Metcalfe CR 1960. Anatomy of Monocotyledons. Gramineae. Clarendon Press: Oxford; 731.

Munir M, Khan MA, Ahmed M, Bano A, Ahmed SN, Tariq K, Tabassum S, Mukhtar T, Ambreen M, Bashir S. 2011. Foliar epidermal anatomy of some ethnobotanically important species of wild edible fruits of northern Pakistan. Journal of Medicinal plants Research, 5(24): 5873-5880.

Nwokeocha CC. 1996. Foliar Epidermal Studies in Oryza punctata. Nigerian Journal of Botany, 9: 49-58.

Ogie-Oda EA, Mokwenye AI, Kekere O, Timothy O. 2010. Comparative vegetative and foliar epidermal features of three Paspalum L. species in Edo State, Nigeria. Ozean Journal of Applied Sciences, 3(1): 29-38.

Ogundipe OT, Olatunji OA. 1991. Vegetative anatomy of Brachiaria obtussiflora (Hochst. ex A. Rich.) Stapf and Brachiaria callopus (Pilg.) Stapf 
(Poaceae). Feddes Repertorium, 102(23): 159-166.

Sano Y 1980. Adaptive strategies compared between the diploid and tetraploid form of Oryza punctata. Bot. Mag. Tokyo, 93: 171-180.

Srivastava AK. 1978. Study of leaf epidermis in the genus Digitaria Rich (Gramineae). J. Indian Bot. Soc., 57: 155-160.

Stace CA. 1965. Cuticular studies as an aid to plant taxonomy. Bull. of the British Museum (Natural History) Bot., 4: 378.

Stace CA. 1984. The taxonomic importance of the leaf surface. In Current Concepts in Plant Taxonomy, Heywood VH, Moore
DM (eds). Academic Press: London and Orlando.

Thakur HA, Patil DA. 2014. Foliar epidermal studies of plants in Euphorbiaceae. Taiwania, 59: 59-70.

Watanabe N, Fujii C, Shirota M, Furuta Y. 1993. Changes in chlorophyll thykaloid proteins and photosynthetic adaptation to sun and shade environments in diploid and tetraploid Oryza punctata Kotschy and diploid Oryza eichingeri Peter. Plant Physiol. Biochem., 31(4): 469-474. 